\title{
Exonic duplication of the OTC gene by a complex rearrangement that likely occurred via a replication-based mechanism: a case report
}

\author{
Katsuyuki Yokoi ${ }^{1,2}$, Yoko Nakajima ${ }^{1}$, Hidehito Inagaki ${ }^{2}$, Makiko Tsutsumi ${ }^{2}$, Tetsuya Ito $^{1}$ and Hiroki Kurahashi ${ }^{2^{*}}$ (DD
}

\begin{abstract}
Background: Ornithine transcarbamylase deficiency (OTCD) is an X-linked recessive disorder involving a defect in the urea cycle caused by OTC gene mutations. Although a total of 417 disease-causing mutations in OTC have been reported, structural abnormalities in this gene are rare. We here describe a female OTCD case caused by an exonic duplication of the OTC gene (exons 1-6).

Case presentation: A 23-year-old woman with late-onset OTCD diagnosed by biochemical testing was subjected to subsequent genetic testing. Sanger sequencing revealed no pathogenic mutation throughout the coding exons of the OTC gene, but multiplex ligation-dependent probe amplification (MLPA) revealed duplication of exons 1-6. Further genetic analyses revealed an inversion of duplicated exon 1 and a tandem duplication of exons 2-6. Each of the junctions of the inversion harbored a microhomology and non-templated microinsertion, respectively, suggesting a replication-based mechanism. The duplication was also of de novo origin but segregation analysis indicated that it took place in the paternal chromosome.
\end{abstract}

Conclusion: We report the first OTCD case harboring an exonic duplication in the OTC gene. The functional defects caused by this anomaly were determined via structural analysis of its complex rearrangements.

Keywords: Ornithine transcarbamylase deficiency, Exonic duplication, Complex rearrangement, Fork stalling and template switching (FoSTeS), Non-homologous end joining (NHEJ)

\section{Background}

Ornithine transcarbamylase (OTC) is a mitochondrial urea cycle enzyme that catalyzes the reaction between carbamyl phosphate and ornithine to form citrulline and phosphate [1]. Ornithine transcarbamylase deficiency (OTCD) is one of the most common urea cycle disorders [2] with an estimated prevalence of 1 in 14,000-77,000 [1]. The human OTC gene, located on the short arm of the $\mathrm{X}$ chromosome (Xp11.4), is $73 \mathrm{~kb}$ with10 exons and $1062 \mathrm{bp}$ of coding sequence [3-5]. Because OTCD is inherited in an X-linked manner, deficient hemizygous males usually develop this disorder. However, a remarkable feature of OTCD is that a

\footnotetext{
* Correspondence: kura@fujita-hu.ac.jp

2Division of Molecular Genetics, Institute for Comprehensive Medical Science, Fujita Health University, 1-98 Dengakugakubo, Kutsukake-cho, Toyoake, Aichi 470-1192, Japan

Full list of author information is available at the end of the article
}

substantial subset of heterozygous females also develop this condition. The symptoms of carrier females vary in terms of onset and severity. Since the OTC gene is subject to $\mathrm{X}$-inactivation, it is believed that this phenotypic variability depends on a skewed degree of this in the livers of carrier females [5].

In $85-90 \%$ of patients with a biochemical phenotype of OTCD, a mutation can be identified through sequencing or deletion/duplication testing [6]. A total of 417 disease-causing mutations in the OTC gene have been reported to date [1]. Exonic deletions have also been described but no prior case of OTCD caused by exonic duplication has previously been reported [7]. In our current case report, we describe a female patient with OTCD caused by a partial duplication of OTC exons 1-6.

(c) The Author(s). 2018 Open Access This article is distributed under the terms of the Creative Commons Attribution 4.0 International License (http://creativecommons.org/licenses/by/4.0/), which permits unrestricted use, distribution, and reproduction in any medium, provided you give appropriate credit to the original author(s) and the source, provide a link to the Creative Commons license, and indicate if changes were made. The Creative Commons Public Domain Dedication waiver (http://creativecommons.org/publicdomain/zero/1.0/) applies to the data made available in this article, unless otherwise stated. 


\section{Case presentation Patient}

The current study patient was a 23-year-old woman with normal psychomotor development and healthy nonconsanguineous parents. She had frequent episodes of nausea, vomiting, stomachache and temporary elevated transaminase from about 4 years of age. Ammonia and plasma amino acid levels were measured when she was 5 years old. Her serum ammonia was $220 \mu \mathrm{g} / \mathrm{dl}$ (normal range 12 $\sim 60 \mu \mathrm{g} / \mathrm{dl}$ ) and she showed high levels of glutamine (1212 nmol/ml; normal value, 420-700), lower normal limits of citrulline $(18.4 \mathrm{nmol} / \mathrm{ml}$; normal value, 17-43), and lower plasma levels of arginine $(32.2 \mathrm{nmol} / \mathrm{ml}$; normal value, 54-130). A urine metabolic screen indicated a gross elevation in orotate (orotate/creatinine ratio $234.3 \mu \mathrm{mol} / \mathrm{g}$ creatinine; normal value, $4.7 \sim 15.9 \mu \mathrm{mol} / \mathrm{g}$ creatinine). These findings were consistent with OTC deficiency. She was therefore biochemically diagnosed with OTCD and her blood ammonia level has been well controlled since by a protein-restricted diet and by oral sodium phenylbutyrate and arginine. Recently, we performed genetic analysis to identify the genetic alterations of the OTC gene in this patient. However, Sanger sequencing revealed no pathogenic mutation.

\section{Genetic analysis \\ Mutational analyses}

Sanger sequencing was performed to screen for genetic variations at the nucleotide level throughout all coding exons of the OTC gene (Additional file 1). We used UCSC genome browser (http://genome-asia.ucsc.edu/) as human genome assembly. To screen for exonic deletions or duplications, multiplex ligation-dependent probe amplification (MLPA) was performed using the SALSA P079-A3 OTC MLPA kit (MRC Holland, Amsterdam, The Netherlands), in accordance with the manufacturer's recommendations. MLPA products were separated by capillary electrophoresis on an ABI3730 genetic analyzer and then processed using GeneMapper software. The peak heights of the samples were compared with control probes and the ratios of these peaks were calculated for all exons. If the dosage quotient was 1.0, the results were considered normal. Thresholds for deletions and duplications were set at 0.5 and 1.5, respectively.

\section{Quantitative real time $P C R$}

To demarcate the duplicated region, quantitative real-time PCR was conducted on blood DNA from the patient and a male control subject using the Applied Biosystems 7300 real time PCR system (Thermo Fisher Scientific). Several primer pairs were designed for OTC (upstream of exon 1 and intron 6) and RPP30 that was used as an autosomal single copy gene reference to generate amplicons suitable for real-time PCR (Fig. 1b, Additional file 1). The PCR reaction was performed in a $15 \mu \mathrm{L}$ reaction system, containing $2 \mu \mathrm{L}$ of template DNA $(5 \mathrm{ng} / \mu \mathrm{L}), 0.6 \mu \mathrm{L}$ of each primer set $(10 \mu \mathrm{mol} / \mathrm{L}), 0.3 \mu \mathrm{L}$ ROX Reference Dye, $4 \mu \mathrm{L}$ distilled water, and $7.5 \mu \mathrm{L}$ of 2xTB Green Premix Ex TaqII (Tli RNaseH Plus, TaKaRa). Two parallel PCR reactions were prepared for each sample. The amplification cycling conditions were as follows: $95^{\circ} \mathrm{C}$ for $30 \mathrm{~s}$, followed by 40 cycles at $95^{\circ} \mathrm{C}$ $5 \mathrm{~s}$ and $60^{\circ} \mathrm{C}$ for $1 \mathrm{~min}$. Data evaluation was carried out using the 7300 system SDS software and Microsoft Excel. The threshold cycle number $(\mathrm{Ct})$ was determined for all PCR reactions and the same threshold and baseline were set for all samples. The starting copy number of the samples was determined using the $\Delta \Delta \mathrm{Ct}$-Method. $\Delta \Delta \mathrm{Ct}$ method was a modification of the method described in Livak et al. for quantifying mRNA [8]. $\Delta \mathrm{Ct}$ represents the mean $\mathrm{Ct}$ value of each sample and was calculated for OTC and RPP30. The starting copy number of the unknown samples was determined relative to the known copy number of the control sample using the following formula:

$\Delta \Delta \mathrm{Ct}=[\Delta \mathrm{Ct} \mathrm{OTC}($ patient) $-\Delta \mathrm{Ct} \mathrm{RPP} 30$ (patient) $]-[\Delta \mathrm{Ct}$ OTC(female)- $\Delta$ Ct RPP30(female)]. The relative gene copy number was calculated by the expression $2^{-\Delta(\Delta \mathrm{Ct})}$. The starting copy number of male control was also determined as a reference value.

\section{Inverse $P C R$}

Inverse PCR were performed using restriction enzyme TaqI (TaKaRa, Shiga, Japan) to isolate the unknown sequences adjacent to the duplicated region of the OTC gene in the study patient. ApE - A plasmid Editor software was used to identify the recognition sites for the restriction enzyme. The restriction enzyme was chosen based on the following criteria: (1) no cutting of the expected breakpoint area; and (2) endonuclease activity would be unaffected by $\mathrm{CpG}$ methylation of the target sequence. A $100 \mathrm{ng}$ aliquot of genomic DNA from both our patient and a control female was digested with the selected restriction enzyme in a total volume of $30 \mu \mathrm{l}$ at $65^{\circ} \mathrm{C}$ for $90 \mathrm{~min}$. The reaction was inactivated using the QiaQuick PCR Purification Kit. A $20 \mu \mathrm{L}$ sample of digested DNA was then mixed with $23 \mu \mathrm{L}$ of DW, $5 \mu \mathrm{L}$ of $10 \times \mathrm{T} 4$ ligase buffer (TaKaRa, Shiga, Japan) and $2 \mu \mathrm{L}$ of T4 DNA ligase to make a final volume of $50 \mu \mathrm{L}$. Ligation reactions were incubated at $16^{\circ} \mathrm{C}$ for $16 \mathrm{~h}$. For subsequent PCR, $1 \mu \mathrm{L}$ of digested and re-ligated DNA template was used in a total reaction volume of $25 \mu \mathrm{L}$ with Tks Gflex DNA Polymerase (TaKaRa, Shiga, Japan). Primers were designed to avoid repetitive sequences (Additional file 1). The PCR conditions were as follows: 30 cycles of $10 \mathrm{~s}$ at $98^{\circ} \mathrm{C}, 15 \mathrm{~s}$ at $60^{\circ} \mathrm{C}$, and $1 \mathrm{~min}$ at $68^{\circ}$ C. Amplified products were analyzed by gel 


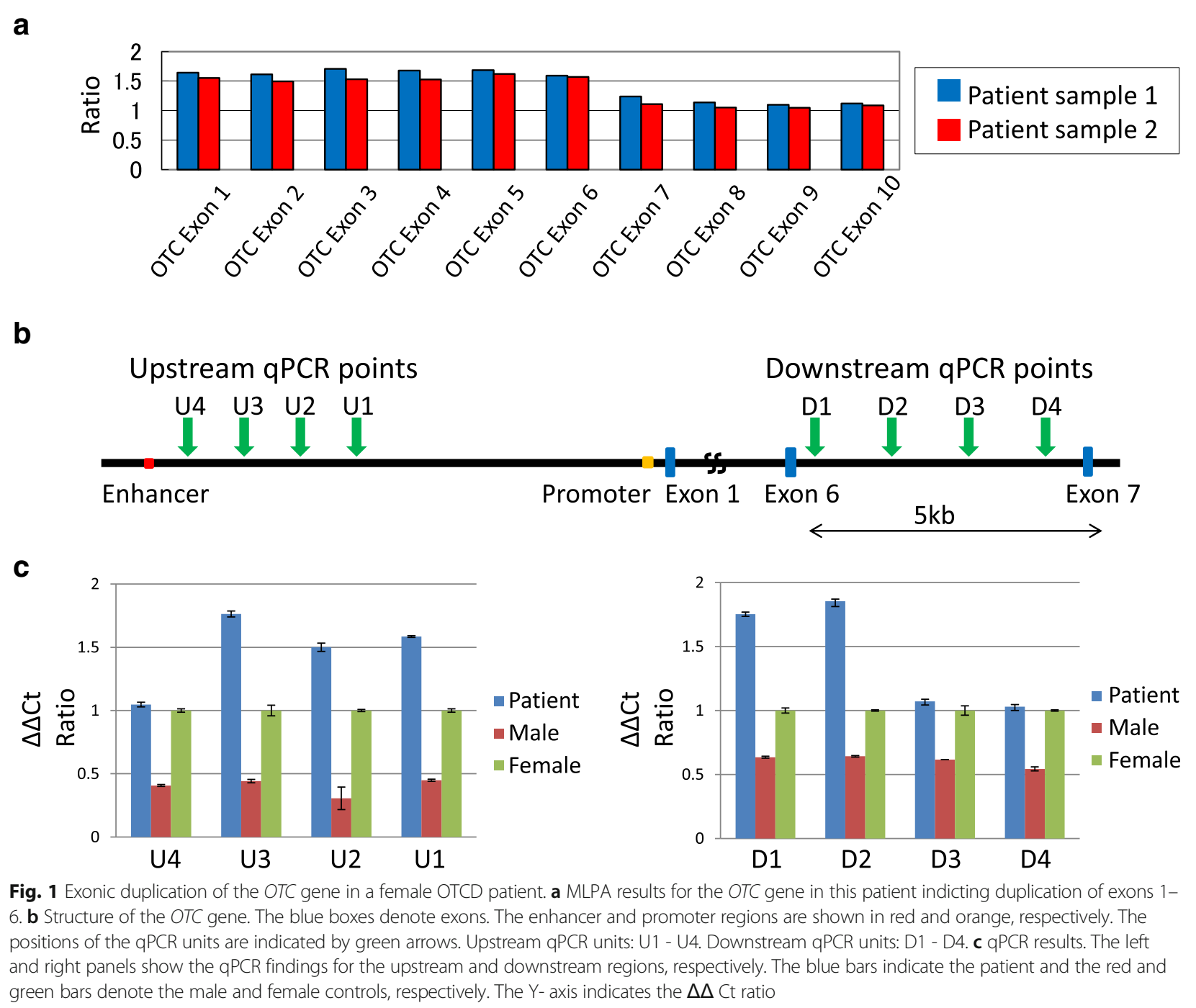

electrophoresis and were purified following nested PCR (Additional file 1). The purified PCR products were sequenced via the standard Sanger method.

\section{Breakpoint analysis on the other side}

PCR was performed using Tks Gflex (TaKaRa, Shiga, Japan) to confirm the other side of the breakpoint sequence. Primer $\mathrm{R}$ which was previously designed for real-time PCR analysis of OTC upstream of exon 1 (i.e. OTC intron 1) was used as primer $\mathrm{F}$ in this reaction (Additional file 1). The PCR conditions and Sanger methodology were similar to those described above.

MLPA revealed the duplication of exons 1-6 of the OTC gene in our current study patient (Fig. 1a). We determined the range of the duplication using quantitative real-time PCR (Fig. 1b). We designed four qPCR experiments (U1-U4) between the promoter and enhancer regions to identify the upstream breakpoint. Likewise, we designed four qPCR assays (D1-D4) within intron 6 to identify the downstream breakpoint. In contrast to the male or female controls that showed $\Delta \Delta \mathrm{Ct}$ ratios of 0.5 or 1.0, respectively, the patient's samples showed a $\Delta \Delta \mathrm{Ct}$ ratio $>1.5$ in some of these qPCR assays, suggesting that these regions were duplicated in this patient (Fig. 1c). The results indicated that the putative upstream breakpoints were located between PCR U3 and U4, and that the downstream breakpoints were between PCR D2 and D3.

We next performed inverse PCR to analyze the genomic structure of the duplicated region. TaqI-digested DNA was used as a template to produce a $3.5 \mathrm{~kb}$ PCR product when amplified with inversely oriented intron 6 primers (Fig. 2a, c). However, an additional small PCR product was detected by agarose gel electrophoresis in the patient sample (Fig. 2a). The amplified products were sequenced after nested PCR (Fig. 2a). As expected, 
a

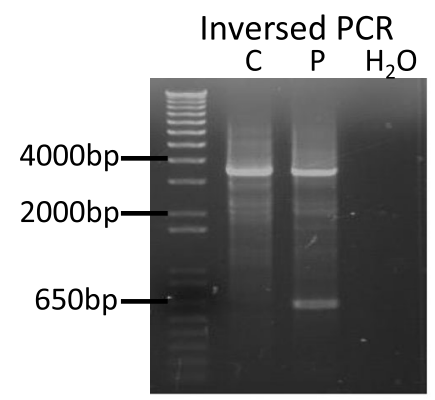

b

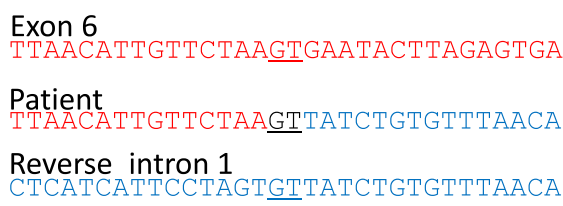

Exon 6

Patient

Reverse intron 1

CTCATCATTCCTÄGTGTTATCTGTGTTTAACA

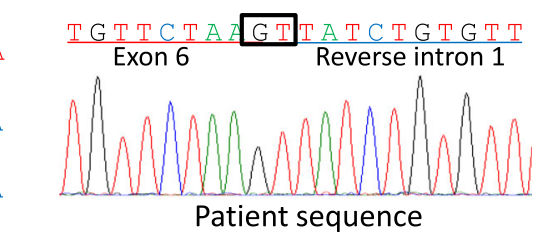

$\stackrel{\rightarrow}{ }$ Primers

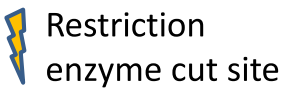

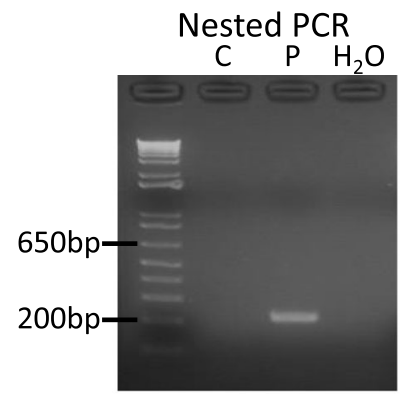

Rearrangement
breakpoit
C

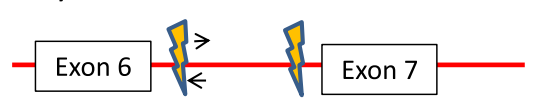

Patient rearrangement

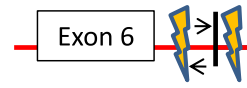

โ $\operatorname{uox} \exists$

Fig. 2 Identification of the duplication junction via inverse PCR. a Isolation of the junction fragment. Two distinct inverse PCR products were observed following agarose gel electrophoresis. The larger product was derived from a normal allele and the small product from a rearranged allele (left). The amplified products were purified following nested PCR (right). P, patient; $\mathrm{C}$, control; $\mathrm{H}_{1} \mathrm{H}_{2} \mathrm{O}$. $\mathbf{b}$ Sanger sequencing of the PCR products including the junction. The unknown sequence next to the junction was identified as intron 1 of the OTC gene in the reverse orientation. The normal exon 6 and intron 1 sequences are aligned in red and blue typeface, respectively. Underlined nucleotides indicate microhomology at the breakpoint junction. c Predicted structure of the junction. Horizontal arrows indicate the recognition sites of the primers used for inverse PCR and the vertical arrows denote the Taql restriction sites

the breakpoint was located within intron 6 (Fig. 2b, c). Unexpectedly however, this breakpoint was found to be connected with intron 1 of the OTC gene in the reverse orientation. The breakpoint junction contained 2 nucleotides of microhomology at the fusion junction (Fig. 2b).

The other side breakpoint was analyzed using standard PCR with primers for the upstream breakpoint region and the breakpoint region in intron 1 . The primer pair amplified only products from the patient's DNA (Fig. 3a). By Sanger sequencing, the upstream region of the OTC gene was found to make an inverted connection with 1 (Fig. 3b, c). This breakpoint junction contained an additional 4 nucleotides (ACTA) of unknown origin (Fig. $3 \mathrm{~b})$. The positions of the two breakpoints in intron 1 were found to be chrX: 38365292 and chrX: 38366694 , which were $1402 \mathrm{bp}$ apart (Fig. 3c). We performed the same PCR amplification of both junctions in the patient's parents but detected no products, suggesting that this complex rearrangement arose de novo. The patient's duplicated region included a common single nucleotide variant (rs752750694, NM_000531.5:c.-844C > T). The patient's father carries an A whereas the mother carries a $\mathrm{G} / \mathrm{G}$ at this site (Fig. 3d). The patient was found to be an $\mathrm{A} / \mathrm{G}$ heterozygote, but the peak of the A nucleotide was two-fold greater than the G-peak, suggesting that the patient carries two copies of A. These data suggest that the de novo duplication was of paternal origin.

\section{Discussion and conclusions}

We here report the first documented case of OTCD caused by an exonic duplication of the OTC gene. Although the MLPA results for this case indicated a simple duplication of exons 1-6, further analysis indicated that it resulted from complex rearrangements. Two possible mechanisms have been proposed for such rearrangements: one is chromothripsis that is caused by chromosome shuttering followed by reunion, and the other is chromoanasynthesis that is a replication-based mechanism also known as fork stalling and template switching (FoSTeS)/microhomology-mediated break-induced replication (MMBIR). According to the replication-based model, the active replication fork can stall and switch templates using complementary template microhomology to anneal and prime DNA replication. This 
a

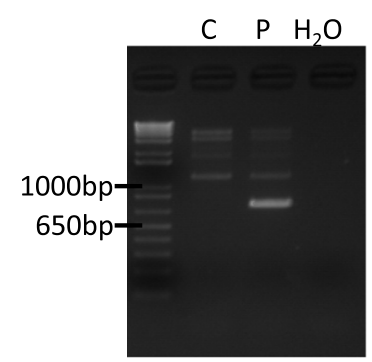

b

Reverse upstream of exon 1

CAGGAAGGAGGTCACTTTGGGAAAGGGGTGTTACCG Patient

CAGGAAGGAGGTCACACTAGTTGTGTGACTAGTAAG Intron 1

GGTAAATAAACTGCCCTAGGTTGTGTGACTAGTAAG

C Putative rearrangement
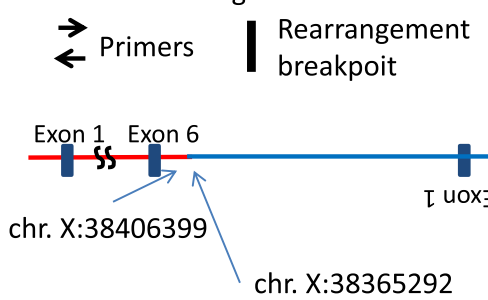

d

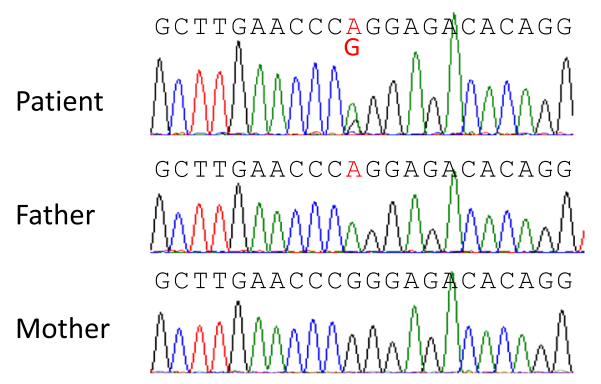

G G A G G T C A A C T A T T T T G T G

Reverse upstream Insertion Intron 1

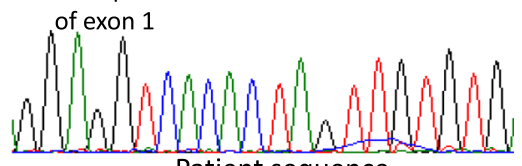

Patient sequence

Fig. 3 The complex rearrangement in the OTC gene of the study patient likely occurred via a replication-based mechanism. a Identification of the other junction by PCR. The PCR primer pair successfully amplified the junction product only from the OTCD study patient DNA. P, patient; $C$, control; $H$, $\mathrm{H}_{2} \mathrm{O}$. b Sanger sequencing results for the PCR products including the junction. The normal sequence of the OTC gene upstream region and intron 1 are aligned in blue and red typeface, respectively. Underlined bases denote a non-templated microinsertion at the junction. c Predicted structure of the complex rearrangement leading to the OTC gene duplication. The positions of the PCR primers are indicated by black arrows. The first junction is indicated by a blue arrow. The nucleotide position of the breakpoints on the X-chromosome are also indicated. The position of the two breakpoints in intron 1 were found to be chr. X: 38365292 and chr. X: 38366694, which were 1402 bp apart. d Trio-genotyping of the common single nucleotide variant (rs752750694, NM 000531.5:C.-844C > T). The patient's father carries A and her mother carries G/G. The patient was found to be an A/G heterozygote, but her A peak was two-fold higher than the G-peak. The areas under the curve (AUC) were 900 (A) and 328 (G) (Image J)

mechanism enables the joining or template-driven juxtaposition of different sequences from discrete genomic positions and can result in complex rearrangements [9].

In our current OTCD case, one junction presented 2 nucleotides of microhomology (GT), and the other junction manifested 4 nucleotides as a microinsertion (ACTA). Copy number variation with complex rearrangements and the presence of microhomology is indicative of a replication-based mechanism but the evidence for a non-templated microinsertion is noteworthy. Microinsertions are often observed in non-proofing DNA repair processes such as non-homologous end joining (NHEJ), which is activated by double-strand breaks [10]. However, a considerable body of evidence now suggests that microinsertions can be identified at junctions mediated by DNA replication-based mechanisms $[11,12]$. A recent study has also suggested that an NHEJ-like pathway mediated by $\operatorname{Pol} \theta$, which is an alternative NHEJ mechanism, may be induced by replication stress [13]. Taken together, an alternative NHEJ pathway might be activated during aberrant replication to restore DNA integrity, thus leading to chromoanasynthesis.

The evidence to date also suggests that de novo mutations occur more frequently in paternal alleles [14]. This bias is attributed to the higher number of DNA replication events in spermatogenesis than in oogenesis. Likewise, chromosomal structural variations are more frequently derived from the father [15]. The complex genomic rearrangements in our present patient were found to be of de novo origin but genotyping of a single nucleotide variant in the OTC gene demonstrated that the rearrangement allele originated from her father. Given the higher chance of the DNA replication errors during spermatogenesis, it might also reflect the replication-based mechanism.

MLPA can be used in the molecular diagnosis of several genetic diseases whose pathogenesis is related to the presence of deletions or duplications of specific genes [16]. Although deletions are clearly pathogenic, this is less certain in the case of duplications. In case of the 
OTC gene for example, duplications of the entire gene are innocuous and present as a normal variant in the general population [7]. In cases of partial duplication as seen in our current patient, gene function may not be necessarily be affected when the additional sequence is inserted into another genomic locus. Even in cases of a tandem duplication, it is feasible that one copy of the OTC gene may maintain an intact structure. In the current OTCD case, the inversion of exon 1 occurred together with its duplication. We predicted in this instance that this complex rearrangement would generate a tandem duplication of exons 2-6 and the production of truncated OTC proteins with defective function due to a frameshift or null protein expression due to nonsense-mediated mRNA decay. The functional defects caused by this mutant allele were therefore the cause of the OTCD in this woman.

In conclusion, we report the first case of OTCD caused by a complex rearrangement resulting in exonic duplication of the OTC gene. Our present report also emphasizes the necessity of fully investigating whether pathogenicity has resulted from a genomic duplication.

\section{Additional file}

Additional file 1: PCR primers and genomic coordinates. (a) Primers for Sanger sequences of OTC exons. (b) Primers for qRT-PCR. (c) Other PCR. (XLSX $13 \mathrm{~kb})$

\section{Abbreviations}

FoSTeS: Fork stalling and template switching; MLPA: Multiplex ligationdependent probe amplification; MMBIR: Microhomology-mediated breakinduced replication; NHEJ: Non-homologous end joining; OTC: Ornithine transcarbamylase; OTCD: Ornithine transcarbamylase deficiency

\section{Acknowledgements}

We thank the patient and her family for their participation in this study. We also thank past and present members of our laboratory.

\section{Funding}

No funding was received.

\section{Availability of data and materials}

All data generated or analysed during this study are included in this published article [and its Additional files].

\section{Authors' contributions}

KY did most of the experiments, retrieved the data, drafted and revised the manuscript. YN and TI discovered the patients and provided many data. HI and MT supported and supervised experiments. HK have contributed equally to the manuscript. All authors contributed to and reviewed the manuscript. All authors read and approved the final manuscript.

\section{Ethics approval and consent to participate}

All procedures followed were in accordance with the ethical standards of the responsible committee on human experimentation (institutional and national) and with the Helsinki Declaration of 1975, as revised in 2005(5). The study protocol was approved by the Ethical Review Board for Human Genome Studies at Fujita Health University.

\section{Consent for publication}

Written informed consent to publish medical information and images was obtained from all patients reported in this publication.

\section{Competing interests}

The authors declare that they have no competing interests.

\section{Publisher's Note}

Springer Nature remains neutral with regard to jurisdictional claims in published maps and institutional affiliations.

\section{Author details}

'Department of Pediatrics, Fujita Health University School of Medicine, Toyoake, Japan. ${ }^{2}$ Division of Molecular Genetics, Institute for Comprehensive Medical Science, Fujita Health University, 1-98 Dengakugakubo,

Kutsukake-cho, Toyoake, Aichi 470-1192, Japan.

Received: 13 September 2018 Accepted: 3 December 2018

Published online: 12 December 2018

\section{References}

1. Caldovic L, Abdikarim I, Narain S, Tuchman M, Morizono H. Genotypephenotype correlations in ornithine Transcarbamylase deficiency: a mutation update. J Genet Genomics. 2015;42(5):181-94.

2. Seminara J, Tuchman M, Krivitzky L, Krischer J, Lee HS, Lemons C, et al. Establishing a consortium for the study of rare diseases: the urea cycle disorders consortium. Mol Genet Metab. 2010;100(Suppl 1):S97-105.

3. Lindgren V, de Martinville B, Horwich AL, Rosenberg LE, Francke U. Human ornithine transcarbamylase locus mapped to band Xp21.1 near the Duchenne muscular dystrophy locus. Science. 1984;226(4675):698-700.

4. Horwich AL, Fenton WA, Williams KR, Kalousek F, Kraus JP, Doolittle RF, et al. Structure and expression of a complementary DNA for the nuclear coded precursor of human mitochondrial ornithine transcarbamylase. Science. 1984;224(4653):1068-74

5. Yorifuji T, Muroi J, Uematsu A, Tanaka K, Kiwaki K, Endo F, et al. Xinactivation pattern in the liver of a manifesting female with ornithine transcarbamylase (OTC) deficiency. Clin Genet. 1998;54(4):349-53.

6. Jang YJ, LaBella AL, Feeney TP, Braverman N, Tuchman M, Morizono $H$, et al. Disease-causing mutations in the promoter and enhancer of the ornithine transcarbamylase gene. Hum Mutat. 2018;39(4):527-36.

7. Shchelochkov OA, Li FY, Geraghty MT, Gallagher RC, Van Hove JL, LichterKonecki $U$, et al. High-frequency detection of deletions and variable rearrangements at the ornithine transcarbamylase (OTC) locus by oligonucleotide array CGH. Mol Genet Metab. 2009;96(3):97-105.

8. Livak KJ, Schmittgen TD. Analysis of relative gene expression data using real-time quantitative PCR and the 2(-Delta Delta C(T)) method. Methods. 2001;25(4):402-8.

9. Zhang F, Carvalho CM, Lupski JR. Complex human chromosomal and genomic rearrangements. Trends Genet. 2009a;25(7):298-307.

10. Lieber MR. The mechanism of human nonhomologous DNA end joining. J Biol Chem. 2008;283(1):1-5

11. Hastings PJ, Lupski JR, Rosenberg SM, Ira G. Mechanisms of change in gene copy number. Nat Rev Genet. 2009;10(8):551-64.

12. Zhang F, Khajavi M, Connolly AM, Towne CF, Batish SD, Lupski JR. The DNA replication FoSTeS/MMBIR mechanism can generate genomic, genic and exonic complex rearrangements in humans. Nat Genet. 2009b;41 (7):849-53.

13. Masset $\mathrm{H}$, Hestand MS, Van Esch $\mathrm{H}$, Kleinfinger P, Plaisancié J, Afenjar A, et al. A distinct class of Chromoanagenesis events characterized by focal copy number gains. Hum Mutat. 2016;37(7):661-8.

14. Jónsson H, Sulem P, Kehr B, Kristmundsdottir S, Zink F, Hjartarson E, et al. Parental influence on human germline de novo mutations in 1,548 trios from Iceland. Nature. 2017;549(7673):519-22.

15. Kurahashi $\mathrm{H}$, Bolor $\mathrm{H}$, Kato $\mathrm{T}$, Kogo $\mathrm{H}$, Tsutsumi $\mathrm{M}$, Inagaki $\mathrm{H}$, et al. Recent advance in our understanding of the molecular nature of chromosomal abnormalities. J Hum Genet. 2009;54(5):253-60.

16. Stuppia L, Antonucci I, Palka G, Gatta V. Use of the MLPA assay in the molecular diagnosis of gene copy number alterations in human genetic diseases. Int J Mol Sci. 2012;13(3):3245-76. 